\title{
Aerial features removing from Ground Penetrating Radar profiles
}

\author{
Guillaume Boutard, Christian Camerlynck, Michel Dabas \\ Département de Géophysique Appliquée, Université Paris VI \\ Marc Descloîtres, Henri Robain \\ Centre ORSTOM Mle-de France, Bondy
}

\section{Theory}

The Ground Penetrating Radar (GPR) has demonstrated its particular ability for many sub-surface investigations. Nevertheless, even in the case of an optimal response from the ground, the image is sometimes disturbed by unwanted features (Sun and Young, 1995). Data processing is then a mandatory way to enhance the useful information inside the radargram. Aerial features are one of the noises encountered using non-shielded antennas.

Among other signal processings, F-K filtering applied on CMP gathers could efficiently allow the removing of aerial features and usually need multifold GPR data set. However, we have to keep in mind that multifold surveys need a large amount of time during acquisition and processing, and many trial-and-error for minimal improvement.

Various reflectors such as trees, poles or walls nearby the survey area could generate hyperbolae features which could hide some parts of the section and disrupt reflectors continuity at identical propagation time. An air-velocity migration process should in theory provide an effective way to transform a hyperbola into a point, but in practice considerably alters the whole section. In order to attenuate or totally remove the aerial features from the radargram we need to imagine another way in filtering the data.

We propose a three-steps processing. The first step is to point out apexes of hyperbolae along radar profiles. In most cases visual recognition of hyperbolae pattern is straight forward. If some doubt occurs, an air-velocity migration $\left(v_{\text {air }}=0.3 \mathrm{~m} . n s^{-1}\right.$ ) provides a good way of identifying the hyperbola apex. An instantaneous envelope algorithm could enhance the migration result.

The second step uses a Normal Move-Out correction (Young et al, 1995). We developed an interactive software to flatten hyperbolae using the following time correction:

$$
\mathrm{dt}=\sqrt{4\left(\mathrm{x}-\mathrm{x}_{0}\right) / \mathrm{v}_{\mathrm{air}}{ }^{2}+\mathrm{t}_{0}{ }^{2}}-\mathrm{t}_{0}
$$

for each trace where $x_{0}$ and $t_{0}$ are respectively the spatial and temporal coordinates of the hyperbola apex, and $x$ the current position of the trace. 
The final step consists in applying a spatial high-pass filter after the flattening, choosing the lowest spatial cut-off frequency according to the length of the flattened hyperbola. We then apply a correction using the inverse formula from (1).

But the operator should care about the time range used over the whole profile : the repetition of this processing for each hyperbola would enhance or even create spurious reflectors. In order to minimize this effect, we have to apply this filter over a window restricted in time : the temporal width of this window is chosen as a multiple of the wave length according to the amplitude of the hyperbolic signal. Processing 2 and 3 are performed successively for each hyperbola.

The software stores all the user-defined apex coordinates from the selected hyperbolae and operates steps 2 and 3 . We have shown that it is not necessary to pick very accurately hyperbola apex. This procedure leads to a fast removal of scattering events.

\section{Case study}

A radar profile was acquired along a track in the Nsimi watershed located in the tropical rain forest of south Cameroon, using unshielded $50 \mathrm{MHz}$ antennas. Among many others, two hyperbolae appear to be created by two trees individually located on field, and mask somehow the continuity of reflectors (figure 1). Two gray triangles indicate each apex.

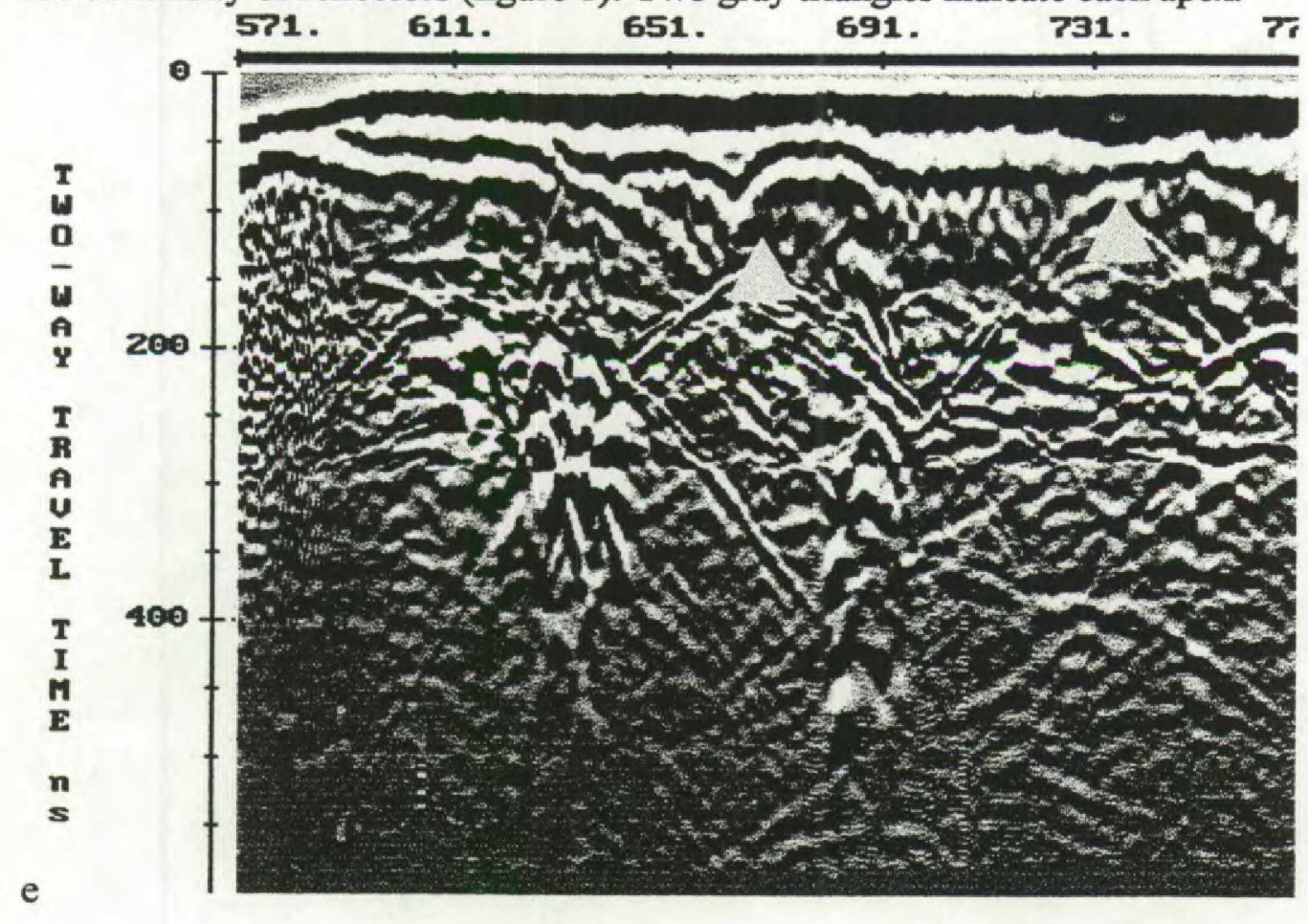

Figure 1

The processing described in the previous part is used to remove both hyperbolae (result shown on figure 2). 


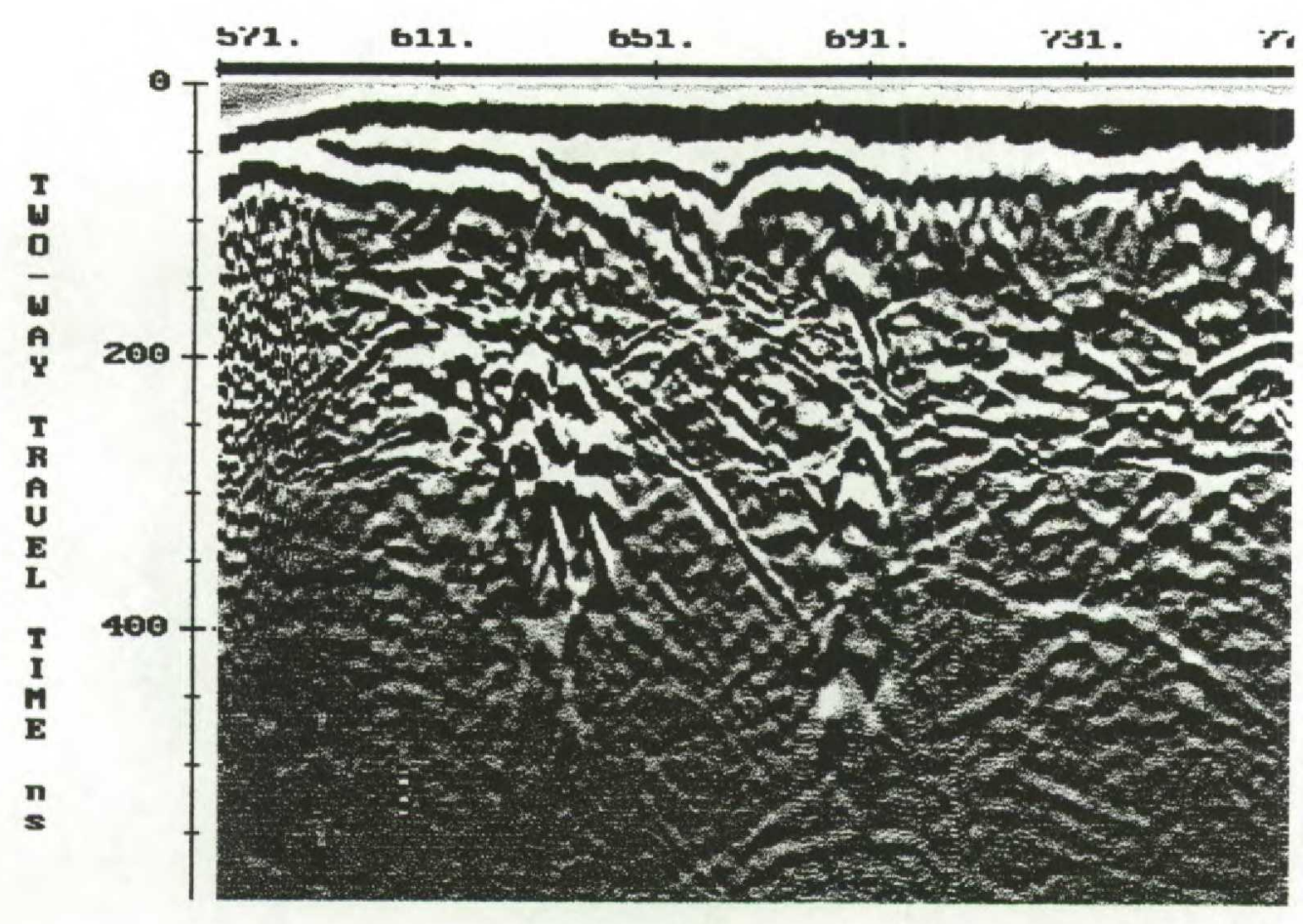

Figure 2

A real enhancement of the image is noticed. Hyperbolae are quite totally erased and provides a more continuous image of the deeping reflector.

This processing could be used to attenuate or eliminate aerial features from data set acquired with unshielded low-frequency GPR antennas.

\section{References:}

Sun, J., Young, R.A., 1995. Recognizing surface scattering in ground-penetrating radar data. Geophysics, 60, 1378-1385.

Young, R.A., Deng, Z., Sun, J., Interactive processing of GPR data. The Leading edge, april 1995, 275-280. 
